\title{
Software para determinar costos de producción en cultivos transitorios de la costa central del Perú
}

Software to determine the production costs of seasonal crops in the central coastal areas of Peru

Vicente Luis Rojas Rojas ${ }^{1}$ y Lorenzo Moisés Ayora Garagate ${ }^{2}$

\section{RESUMEN}

Los objetivos fueron: Obtener software para calcular los costos de producción de un cultivo transitorio por hectárea; crear una base de datos con los insumos, actividades agrícolas e información escrita y verbal del ámbito agrícola de la costa central; y validar el software obtenido a través de una encuesta de satisfacción de usuarios a estudiantes y docentes universitarios afines al agro. Permite determinar la densidad de plantas por hectárea, la cantidad en sacos de la formula NPK, el costo de insumos actualizado, el costo de inversión y la utilidad para una hectárea de un cultivo. El software considera el ingreso; usuario-contraseña; presentación inicial; datos generales para registrar un cultivo; datos específicos para registrar el cultivo definido; revisión, corrección y/o incorporación de datos de los costos directos e indirectos; análisis económicos, índice de rentabilidad, reporte final en soles o dólares, e imprime la hoja del costo de producción por hectárea. Se concluyó que el $98 \%$ de los encuestados afirmaron que el software es amigable y el $84 \%$ de los encuestados calificaron como un programa satisfactorio a los requerimientos de los usuarios dedicados al agro. El software es amigable, fácil de usar y brinda apoyo adecuado a los usuarios.

Palabras clave: actividades agrícolas; base de datos; rendimientos; software agrícola.

\footnotetext{
Universidad Nacional Agraria la Molina. Lima, Perú.

2 Universidad Nacional Santiago Antúnez de Mayolo. Huaraz, Perú.

(C) Los autores. Este artículo es publicado por la Revista Aporte Santiaguino de la Universidad Nacional Santiago Antúnez de Mayolo. Este es un artículo de acceso abierto, distribuido bajo los términos de la Licencia Creative Commons Atribución-NoComercial-CompartirIgual 4.0 Internacional. (http://creativecommons.org/licenses/ by-nc-sa/4.0/), que permite el uso no comercial, distribución y reproducción en cualquier medio, siempre que la obra original sea debidamente citada.
} 


\begin{abstract}
The main objectives were: to program a software to calculate the costs of seasonal crop production per hectare; to create a database of inputs, written and verbal agriculture technical information for the central coastal areas, and to validate the software surveyed in a user satisfaction tested to students and academic staff involved into agriculture. It allow users to determine the sowing rate per hectare, number of fertilizer bags according to NPK formulas, the current input costs, investment costs and profitability in one cropping hectare. The software considered the income, user password, initial presentation, general information to register a crop, record of specific data defined by agriculture tasks, review, correction or adding data from direct and indirect costs, economic analysis, rate of return, final report in local currency (soles) or dollars, and printing the sheet production costs per hectare. It was concluded that $98 \%$ of respondents said that the software was friendly and $84 \%$, it was a successful program to fill the needs of users engaged in agriculture. The software is friendly, easy to use and provides adequate support to users.
\end{abstract}

Keywords: agricultural activities; database; performances; agricultural software.

\title{
INTRODUCCIÓN
}

La agricultura en la costa del Perú se realiza geográficamente en una faja angosta de territorio surcada por 53 valles que vierten su aguas en el océano Pacífico. Los valles contienen una diversidad de sistemas de cultivo y producción expresados en las cédulas de cultivos, los niveles tecnológicos y productivos. A pesar de esta diversidad es posible identificar la parte media de la Costa Central del Perú constituida por la porción costera de los departamentos de Lima e Ica, como el área privilegiada porque tiene un régimen regular de agua de riego proveniente de 17 ríos durante todo el año. Esta regularidad permite lograr una producción estable de cultivos transitorios, que ocupan los primeros lugares en superficie sembrada, rendimientos y producción a nivel nacional, principalmente en los cultivos de maíz (Zea mays L.), algodón (Gossypium barbadense L.) y papa (Solanum tuberosum L.) (Eguren, 2003).

Los costos de producción de los cultivos en cada valle están relacionados a los insumos utilizados por unidad de área. Cada valle tiene una cédula de cultivo que converge en cultivos permanentes y transitorios o estacionales. Igualmente, los productores disponen de ambos grupos de cultivos dentro de sus parcelas o propiedades. En general, la estructura de costos pueden ser similares, pero las diferencias serán en el uso o no y en las cantidades de los insumos para generar la producción. Aunque los productores 
puedan usar diferentes unidades de áreas en función de la región, en el presente trabajo la unidad de área usada ha sido la hectárea.

En la actual era de la globalización y desarrollo tecnológico urge la necesidad de disponer de programas informáticos o software para el sector agrícola que facilite la toma de decisiones a los productores del campo. Un software especializado debe ser el mejor aporte para la educación y tecnología agrícola como herramienta práctica en la elaboración de base de datos, la estructura de gastos y costos de producción de los cultivos. La poca o nula creación local de software especializado para la enseñanza, investigación y extensión agrícola constituye una gran limitante para el desarrollo eficaz de estas actividades y cuyo potencial requiere urgente explotación de acuerdo a la realidad nacional. El uso de software en las computadoras ayudará en la supervivencia y desarrollo de una empresa o unidad agrícola, que debe enfrentar condiciones de mercado cada vez más exigentes.

Los objetivos del presente trabajo fueron: (1) Crear una base de datos con los insumos, actividades agrícolas e información escrita y verbal proveniente de los productores del ámbito agrícola de la Costa Central; (2) Desarrollar un software para calcular los costos de producción de un cultivo transitorio por hectárea; y (3) Validar el software obtenido a través de una encuesta de satisfacción de usuarios a estudiantes y docentes universitarios afines al agro.

El software del computador es el conjunto de instrucciones a ser utilizado en un soporte legible -máquina- capaz de procesar información en forma automática (Pressman, 2010). La asignación del costo al producto, se hace combinando los gastos incurridos en forma directa, con los gastos de otros procesos o actividades relacionadas con la producción. Los elementos que forman el costo de un artículo bajo este sistema son: materia prima, mano de obra, gastos directos e indirectos de fabricación que pueden ser variables o fijos (Sierra, 2011). Agricostos, software destinado a la "industria agrícola" permite gestionar (administrar) los costos reales derivados del proceso productivo de una hacienda o fundo, tales como costos de mano de obra (personal), aplicación de insumos, uso de maquinarias, riegos y gastos diversos (Agricostos, 2009).

AgroWin, software diseñado para el sector agropecuario, facilita la gestión contable y administrativa de los recursos. Permite maximizar los ingresos y minimizar los costos a través del afianzamiento de la labor administrativa. AgroWin es un software seguro, versátil y de fácil manejo en el mercado. AgroWin Móvil es un complemento para AgroWin, que permite a los usuarios conectarse al servidor de datos central de AgroWin y realizar ciertas actividades de gestión y consulta desde dispositivos móviles como celulares o tabletas (AgroWin, 2017) 


\section{MATERIALES Y MÉTODOS}

El presente trabajo se realizó en el Centro de Cómputo de la Escuela de Posgrado de la Universidad Nacional Agraria La Molina (UNALM), Lima, Perú. La información fue procesada utilizándose Visual FoxPro versión 6.0, MS-Excel versión 2003, MSWord versión 2003 e Internet Explorer versión 6.0. Un programador con experiencia en trabajos vinculados al desarrollo de programas agropecuarios asesoró al equipo de investigadores en trabajo informático.

La Base de Datos fue creada y desarrollada en fases mediante la (a) Recopilación de información; (b) Estructuración de los campos e interrelación con la aplicación del software y (c) el Mantenimiento de la Base de Datos.

El procedimiento para la obtención del Software fue desarrollado en seis fases o ciclos de vida (Senn, citado por Urbina, 2002): en la fase de (a) Análisis, el trabajo consistió en identificar la necesidad y requerimiento de implementar un software; en el (b) Diseño, el equipo trabajó en el refinamiento de la representación arquitectónica, que lleva una estructura detallada de datos y las representaciones algorítmicas del software; en la fase de (c) Codificación, las especificaciones del diseñó en código fuente fueron traducidas mediante la aplicación de un lenguaje de programación; (d) la Prueba estuvo relacionada a la revisión final de las especificaciones del diseño y de la codificación; en la (e) Implementación, el software fue instalado en las computadoras, verificando el correcto funcionamiento para que no ocurran conflictos durante la ejecución, adaptándose y mejorándose el programa de acuerdo a las sugerencias del usuario (encuesta) y, finalmente, la fase de (f) mantenimiento fue integral y preventivo del software.

El procedimiento para la Validación consistió en elaborar la "Encuesta de Satisfacción del Usuario”, aplicándola a una muestra obtenida de una población promedio mensual de asistencia al Centro de Cómputo constituida por estudiantes de la Escuela de Posgrado y docentes afines al agro de la UNALM, la tabulación e interpretación de los resultados obtenidos.

\section{RESULTADOS}

\section{Creación de la Base de Datos}

La información analizada y evaluada permitió crear diez Bases de Datos Específicos (BDE) a partir de la Base de Datos General (BDG). Las BDE creadas fueron denominadas: Cultivos, Lugar, Semillas, Abonos, Riegos, Labores, Control, Rendimiento, Precio e Insumos. El análisis principal estuvo referido a Cultivos e Insumos. La BDE Cultivos (tabla 1) comprendió las columnas o cinco campos correspondientes a la categoría de cultivos, ciclo de vida, nombre del cultivo, tipo o variante de cultivo, y cultivar o variedad. Las filas correspondieron a la información detallada de los cultivos principales de la costa central del Perú. 
Tabla 1. Base de datos de los cultivos transitorios en la costa central del Perú

\begin{tabular}{|c|c|c|c|c|c|c|}
\hline \multirow{2}{*}{$\begin{array}{c}\text { Categoría del } \\
\text { cultivo } \\
\text { (Grupo) }\end{array}$} & \multirow{2}{*}{$\begin{array}{l}\text { Ciclo de } \\
\text { Vida }\end{array}$} & \multirow{2}{*}{$\begin{array}{l}\text { Nombre } \\
\text { común }\end{array}$} & \multicolumn{2}{|c|}{ Tipo (Variante) } & \multicolumn{2}{|c|}{$\begin{array}{l}\text { Cultivar } \\
\text { (variedad) }\end{array}$} \\
\hline & & & $\mathrm{E}$ & $\mathrm{nE}$ & $\mathrm{D}$ & $\mathrm{C}$ \\
\hline Cereales Mayores & Anual & Maíz & 3 & 1 & 68 & 81 \\
\hline Forrajeros & Anual & Maíz & 1 & & 1 & 2 \\
\hline Cereales Menores & Anual & Cebada & & & 1 & 7 \\
\hline Fibra Vegetal & Anual & Algodón & 1 & 1 & 2 & 32 \\
\hline Flores & Anual & Marigold & & & 1 & 8 \\
\hline \multirow{4}{*}{$\begin{array}{l}\text { Leguminosas de } \\
\text { Grano }\end{array}$} & & & & & & 30 \\
\hline & Anual & Frijol & 2 & & 2 & \\
\hline & & Garbanzo & & & 1 & 4 \\
\hline & & Pallar & & & 1 & 5 \\
\hline Oleaginosas & Anual & Maní & & & 1 & 2 \\
\hline \multirow[t]{11}{*}{ Olerizas } & Anual & Ají & 8 & & 1 & 27 \\
\hline & & Arveja & 5 & 1 & 2 & 22 \\
\hline & & Melón & 3 & 1 & & 14 \\
\hline & & Rabanito & & 1 & & 3 \\
\hline & & Sandia & 2 & 1 & & 7 \\
\hline & & Tomate & 3 & 1 & & 17 \\
\hline & & Zapallo & 2 & 1 & & 3 \\
\hline & Bianual & Cebolla & 6 & 2 & 1 & 13 \\
\hline & Bianual & Ajo & & & 1 & 8 \\
\hline & Bianual & Zanahoria & & 1 & & 2 \\
\hline & Multianual & Fresa & & 2 & 1 & 1 \\
\hline \multirow[t]{3}{*}{$\begin{array}{l}\text { Tuberosas y Raí- } \\
\text { ces }\end{array}$} & Anual & Camote & 2 & 1 & & 24 \\
\hline & & Papa & 2 & 4 & & 18 \\
\hline & & Yuca & & 1 & & 5 \\
\hline
\end{tabular}

$\mathrm{E}=$ Especificado; $\mathrm{nE}=$ No especificado; $\mathrm{D}=$ Desconocido $\mathrm{C}=$ Conocido

\section{Desarrollo del software COST-1}

La BDE Insumos proporcionó la información necesaria para el desarrollo del software COST-1. Los datos mensuales de los precios de productos que estaban a la venta en las casas comerciales de las principales ciudades de la costa central (Barranca, Huacho, Huaral, Lima, Cañete, Chincha, Pisco, Ica, etc.) permitieron proyectar los costos de producción reales a la fecha del trabajo o almacenar los costos durante toda la campaña de un cultivo. De esta manera, la tabla 2 fue elaborado a partir de la información de la tabla 1, incluyéndose como nuevas columnas el tipo de insumo, precio, la unidad y el contenido de recipiente de venta. 
Vicente Luis Rojas Rojas y Lorenzo Moisés Ayora Garagate

Tabla 2. Base de datos de insumos usados en los cultivos transitorios en la costa central del Perú

\begin{tabular}{|c|c|c|c|c|c|c|}
\hline Insumo & $\begin{array}{c}\text { Cultivo o Tipo } \\
\text { o Clase }\end{array}$ & $\begin{array}{l}\text { Cultivar o } \\
\text { Variedad* }\end{array}$ & $\begin{array}{c}\text { Precio } \\
\text { S/. o US\$ }\end{array}$ & Unidad & Unidad & $\begin{array}{c}\text { Cantidad } \\
\mathrm{Kg} / \mathrm{ha}\end{array}$ \\
\hline \multirow[t]{15}{*}{ Semilla } & $\begin{array}{c}\text { Maíz Amarillo } \\
\text { Duro }\end{array}$ & 5 & 350 a 425 & Soles & Bolsa & 25 \\
\hline & Cebada & 1 & 6 & Soles & $\mathrm{Kg}$ & \\
\hline & Algodón & 1 & 120 & Soles & Bolsa & 11.5 \\
\hline & Frijol & 2 & 8 & Soles & $\mathrm{Kg}$ & \\
\hline & Ají & 1 & 220 & Dólares & Bolsita & 0.5 \\
\hline & Arveja & 1 & 15 & Soles & $\mathrm{Kg}$ & \\
\hline & Cebolla & 1 & 80 & Dólares & Sobre & 0.5 \\
\hline & Melón & 1 & 385 & Dólares & Lata & \\
\hline & Rabanito & 1 & 9,5 & Dólares & Sobre & \\
\hline & Sandia & 1 & 400 & Dólares & Sobre & \\
\hline & Tomate & 1 & 515 & Dólares & Sobre & \\
\hline & Zanahoria & 1 & 125 & Dólares & Sobre & \\
\hline & Zapallo & 1 & 20 & Dólares & Sobre & 2 \\
\hline & Papa & 1 & 1,1 & Soles & $\mathrm{Kg}$ & 2000 \\
\hline & Camote & 1 & 5 & Soles & Atado & esquejes \\
\hline \multirow[t]{4}{*}{ Abono } & Sintético & 7 & 43 a 145 & Soles & Saco & \\
\hline & Orgánico & 1 & 90 & Soles & Tonelada & \\
\hline & Foliar & 5 & 13,2 a 54 & Soles & Litro & \\
\hline & Foliar & 1 & 19,1 a 22,4 & Soles & Kilo & \\
\hline Aceite & Adherente & 4 & 18 a 165 & Soles & Litro & \\
\hline \multirow[t]{11}{*}{ Pesticidas } & Acaricida & 1 & 190 & Soles & Litro & \\
\hline & Acaricida & 1 & 13 & Soles & Gramo & \\
\hline & Fungicida & 3 & 30 a 40 & Soles & Gramo & \\
\hline & Fungicida & 14 & 20 a 130 & Soles & Kilo & \\
\hline & Fungicida & 13 & 23 a 410 & Soles & Litro & \\
\hline & Herbicida & 1 & 170 & Soles & Galón & \\
\hline & Herbicida & 2 & 43 a 90 & Soles & Kilo & \\
\hline & Herbicida & 9 & 32 a 157 & Soles & Litro & \\
\hline & Insecticida & 8 & 14 a 85 & Soles & Gramo & \\
\hline & Insecticida & 7 & 10 a 240 & Soles & Kilo & \\
\hline & Insecticida & 37 & 38 a 450 & Soles & Litro & \\
\hline \multirow[t]{2}{*}{ Equipos } & Bomba & 1 & 238 & Soles & Unidad & \\
\hline & Pulverizadora & 1 & 350 & Soles & Unidad & \\
\hline
\end{tabular}

* Número considerado en el software

Fuente: Elaboración propia a partir de la información proporcionada por las distribuidoras de productos agrícolas. Lista de precios comerciales. Localidad de Barranca Lima 
La primera versión del software denominado COST-1 versión 1.0 (figura 1) permitió determinar los costos de producción por hectárea de un cultivo de la costa central. El software presenta las siguientes características: en la página inicio incluye un manual, comunicación vía web y el acceso inicial de usuario - contraseña. En la barra de menú la opción "Costos de producción" abre una ventana en la parte central del área de trabajo denominado Datos Generales, donde ingresa el registro de los datos básicos del cultivo, variedad y lugar de producción. Luego, son ingresados el valor de jornal, la hora/máquina y la fórmula de Nitrógeno, Fósforo y Potasio (NPK). El distanciamiento entre surcos, entre plantas y número de semillas son ingresados para obtener automáticamente la densidad de plantas por hectárea. Con la información ingresada, los costos directos e indirectos prácticamente están listos. Finalmente el software COST-1 muestra el costo total, el ingreso total, la utilidad, el índice de rentabilidad y la información está lista para la impresión del reporte completo del costo de producción de un cultivo por hectárea.

El software tiene algunas limitaciones que pueden ser subsanadas en una segunda versión. Entre las limitaciones del software están que no incluye las horas por tracción animal, el valor monetario por jornal y las horas máquina ingresadas por Datos Específicos no es modificable en las actividades dentro del mismo registro. Adicionalmente, el software no tiene agrupada la información por valles en forma estándar con las localidades que la constituyen, tampoco establece costos en función de las condiciones climáticas, ni extracción de nutrientes y fijación radicular de cultivos.

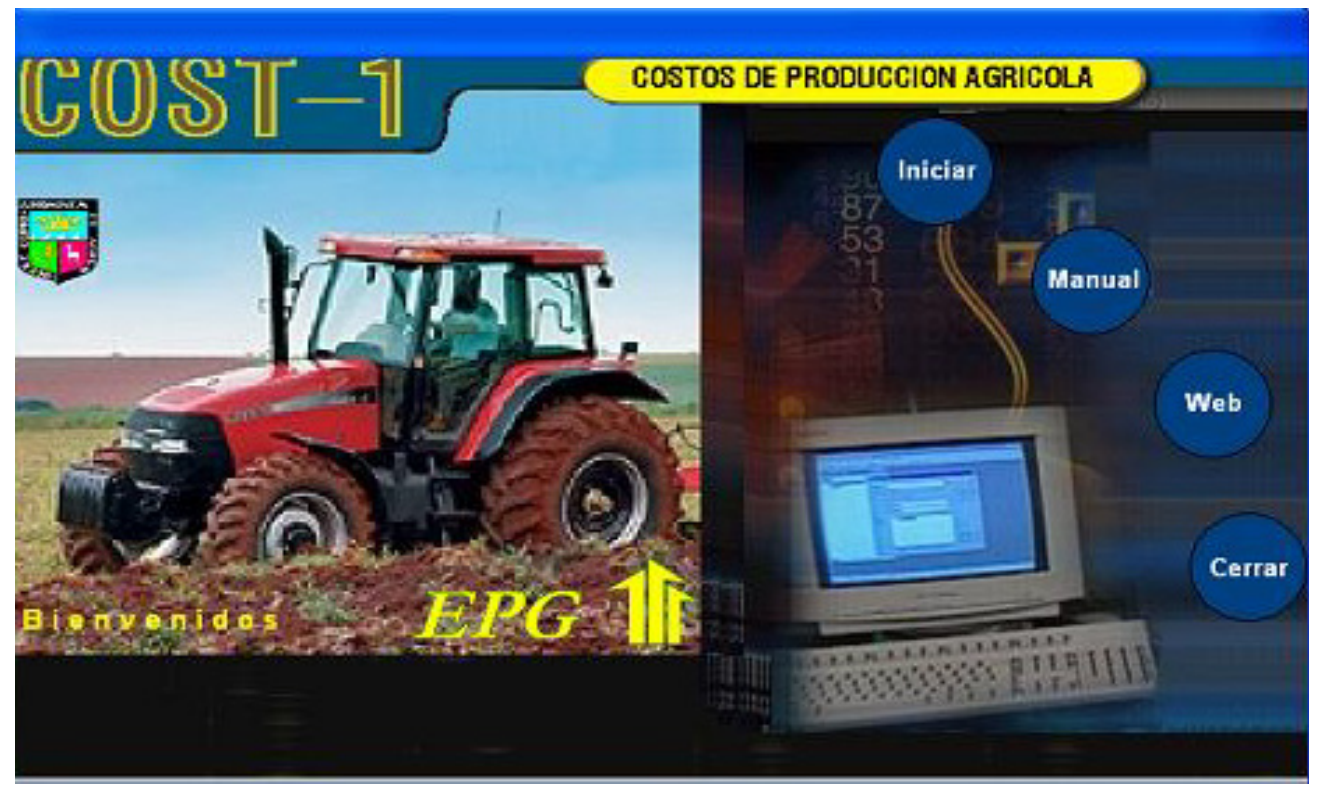

Figura 1. Portada del software COST-1 
Tabla 3. Resultados de la encuesta en base a la experiencia y conocimiento del entrevistado

\begin{tabular}{|c|c|c|c|}
\hline \multicolumn{2}{|c|}{$\begin{array}{l}\text { ¿Tiene experiencia en producción de } \\
\text { cultivos? }\end{array}$} & \multicolumn{2}{|c|}{$\begin{array}{c}\text { ¿Tiene conocimiento de costos de } \\
\text { producción? }\end{array}$} \\
\hline Categoría & $\begin{array}{c}\text { Porcentaje } \\
\qquad(\%)\end{array}$ & Categoría & $\begin{array}{c}\text { Porcentaje } \\
\qquad(\%)\end{array}$ \\
\hline No & 20 & $\mathrm{Si}$ & 68 \\
\hline $\mathrm{Si}$ & 80 & $\begin{array}{l}\text { No } \\
\text { Algo }\end{array}$ & $\begin{array}{c}4 \\
28\end{array}$ \\
\hline
\end{tabular}

\section{Validación del uso del software COST-1}

La validación del software a través de la encuesta aplicada fue favorable en el uso del software. Los resultados mostrados en la tabla 3, indican que el $80 \%$ de los encuestados respondieron haber tenido experiencia en la producción de cultivos y más de dos tercios $(68 \%)$ de los encuestados aseguró conocer sobre los Costos de Producción de Cultivos. Una respuesta esperada si tomamos en cuenta que este grupo pertenece a las personas con experiencia en producción de cultivos.

La respuesta a la pregunta si usaron algún otro medio para elaborar los costos de producción de un cultivo o si conocían algún software sobre el tema, los resultados son mostrados en la tabla 4. En la primera parte de la pregunta, el $44 \%$ de los encuestados indicó que hizo uso de una calculadora y el $30 \%$ de una hoja de cálculo electrónico (MS-Excel). En la segunda parte de la pregunta, el $94 \%$ de los encuestados señalaron no conocer un software que determine Costos de Producción de Cultivos.

Tabla 4. Respuesta por la forma de calcular y conocer software de costos de producción

\begin{tabular}{cc|cc}
\hline $\begin{array}{c}\text { De haber tenido experiencia en costos } \\
\text { ¿Cómo calculó mayormente? }\end{array}$ & $\begin{array}{c}\text { ¿Conoce algún software sobre } \\
\text { Costo de Producción? }\end{array}$ \\
\hline Categoría & Porcentaje & Categoría & Porcentaje \\
A mano sumando y restando & 12 & No & 94 \\
Con una calculadora & 44 & Si & 6 \\
Encargó a otra persona. & 4 & & \\
No tuvo experiencia & 10 & & \\
Otra forma: Con MS-Excel. & 30 & & \\
\hline
\end{tabular}


La validación concluye que el software COST-1 proporciona satisfacción a los usuarios (98 \%, amigable) y una alta aceptación (84\%, Bueno a Muy Bueno) (tabla 5). Esto puede pronosticar que el software COST-1 tendría una alta demanda por los usuarios del agro nacional.

Tabla 5. Respuesta al software Cost-1: Amigable y calificación

\begin{tabular}{|c|c|c|c|}
\hline \multicolumn{2}{|c|}{ ¿El software Cost-1 fue amigable? } & \multicolumn{2}{|c|}{ ¿Qué calificación daría al software Cost-1? } \\
\hline Categoría & $\begin{array}{l}\text { Porcentaje } \\
\qquad(\%)\end{array}$ & Categoría & $\begin{array}{c}\text { Porcentaje } \\
(\%)\end{array}$ \\
\hline $\mathrm{Si}$ & 98 & Muy Bueno & 34 \\
\hline \multirow[t]{3}{*}{ No } & 2 & Bueno & 50 \\
\hline & & Regular & 16 \\
\hline & & Malo & 0 \\
\hline
\end{tabular}

\section{DISCUSIÓN}

Los datos e información de la base de datos cultivos y los otros creados están enmarcados en el sustento de viabilidad del software obtenido. La FAO (s/f), cuenta con una base de datos por cultivo con características de mayor amplitud en relación a nutrientes, rendimientos y por países, respecto a la propuesta de la presente investigación; la Base de Datos de la FAO sirvió como referencia a las Bases de Datos creadas. La FAO da énfasis de la importancia del uso de una base de datos y motiva a alimentar con datos por zonas agro-ecológicas con los principales cultivos de un país específico. Una base de datos para cultivos como de la FAO, o con otras características no se cuenta en la información del Ministerio de Agricultura, ni en otra organización nacional.

El software Cost-1 obtenido para determinar los costos de producción de cultivos transitorios (anuales) está definido por hectárea, mientras que AgroWin (2017) desde la fecha de su creación fines del milenio anterior a la actualidad está concebido como un sistema, o como un conjunto de módulos individuales pero integrados; no solo por hectárea sino, el costeo proporciona en costos de producción de los lotes o cultivos de 
la finca expresadas en costos por hectárea, costos por sitio y costos por kilogramo de producto producido. Además, Cost-1 cuenta con la compatibilidad del MS-Excel para exportar el costo por hectárea obtenido, mientras que AgroWin por lo moderno de la tecnología alcanzada para su desarrollo, su interrelación es tanto sus bases de datos como sus informes son exportables a formatos de uso común como XLS (Excel de Office), XML, HTML, TXT, RTF, Etc.

Los estudios (encuestas) de satisfacción de usuarios sirven para establecer objetivos en la gestión de sistemas como en el software creado COST-1; por tanto, necesario el diseño y elaboración de una encuesta, necesario para identificar áreas a explorar, definir las características del cuestionario, del proceso de construcción y validación del cuestionario, cálculo muestral, trabajo de campo, análisis de resultados y clasificación por grupos (Pérez, 2009); de dicha aplicación se obtuvo en la presente investigación la validación a través de la Encuesta de Satisfacción del Usuario que el 98 \% de los encuestados afirmaron que el software COST-1 es amigable, por su manejo práctico, didáctico y sencillo; y el 84 \% de los encuestados calificaron al software COST-1 como un programa satisfactorio a los requerimientos de los usuarios involucrados con el agro.

\section{CONCLUSIONES}

La base de datos Cultivos, Lugar, Semillas, Abonos, Riegos, Labor, Control, Rendimiento, Precio e Insumos creados contribuirán al almacenamiento de información de los materiales, actividades, conocimientos escritos y verbales del ámbito agrícola de la costa central.

La primera versión del Software COST-1 versión 1.1 obtenida contribuirá a determinar la densidad de plantas por hectárea, la cantidad en sacos de la fórmula NPK, el costo de insumos actualizado, el costo de inversión, utilidad, análisis económicos, índice de rentabilidad, reporte final en soles o dólares, impresión del costo de producción obtenido para una hectárea de un cultivo transitorio de la costa central.

El 98 y el $84 \%$ de los encuestados afirmaron que el software COST-1 es amigable y un programa satisfactorio a sus requerimientos en el agro respectivamente.

Entre las limitaciones del software no incluye horas por tracción animal, no tiene agrupado por valles información estándar con las localidades que la constituyen, no establece costos en función de condiciones climáticas. 


\section{AGRADECIMIENTOS}

Al Centro de Cómputo de la Escuela de Posgrado de la UNALM por el apoyo logístico. A los docentes de la UNALM doctores Arias C., Soplín V. y Fernández J.; Mg.Sc. Chura Ch. por el apoyo durante la fase de culminación. Al programador Jayo G. por su participación activa desde el prototipo hasta la obtención de la última versión del software. A los encuestados por su gentil participación.

\section{REFERENCIAS BIBLIOGRÁFICAS}

Agricostos. 2009. Software de costos. Lima. <http://www.agricostos.com/> [Consulta: 05-05-2016].

AgroWin.2017.ManualBásicodeAgroWin8 <96040575/Manual-Basico-de-AgroWin8> [Consulta: 05-09-2017].

Eguren, Fernando. 2003. La agricultura de la costa peruana. En Debate Agrario Nº 35. CEPES. Lima, 37 p.

Food and Agriculture Organization of the United Nations. s.f. Base de datos de repuesto a nutrientes. Programa de fertilizantes de la FAO. Roma. < http://www.fao.org/statistics/databases/es/ > [Consulta: 27-07-2016].

Pérez Hidalgo, María del Pilar. 2009. Estudios de satisfacción de usuarios. Formulario de contacto. III Jornadas de Bibliotecas del SSPA, Ronda, 25-26 y 27 de mayo. Junta de Andalucia. Sevilla, 24 p.

Pressman, Roger. 2010. Ingeniería del software. Un enfoque práctico. $7 \mathrm{ma}$ ed. McGraw-Hill. México. 777 p.

Sierra, Felipe. 2011. Taller Contabilidad de Costos. Programas de Gestión Pública En la Universidad del Pacifico. Lima. < http://www.slideshare.net/alafito/costos-niif > [Consulta: 20-10-2016].

Urbina Huertas, José. 2002. Diseño y desarrollo de un software para la evaluación nutricional, funcional y económica de hamburguesas. Tesis. Ing. Industrias Alimentarias. UNALM. Lima. 163 p. 
|| Vicente Luis Rojas Rojas y Lorenzo Moisés Ayora Garagate

Recepción: 04/09/2018

Aceptación: 10/11/2018

\section{Correspondencia}

Vicente Luis Rojas Rojas

vicente@lamolina.edu.pe 